\title{
MÉTODOS DE AJUSTE E MODELOS DE SEMIVARIOGRAMA APLICADOS AO ESTUDO DA VARIABILIDADE ESPACIAL DE ATRIBUTOS FÍSICO-HÍDRICOS DO SOLO ${ }^{(1)}$
}

\author{
Natalino Martins Gomes ${ }^{(2)}$, Antônio Marciano da Silva ${ }^{(3)}$, Carlos Rogério de \\ Mello $^{(3)}$, Manoel Alves de Faria ${ }^{(3)}$ \& Polyanna Mara de Oliveira ${ }^{(4)}$
}

\begin{abstract}
RESUMO
Avaliou-se a variabilidade e a estrutura de dependência espacial de atributos físico-hídricos do solo (volume total de poros - VTP, condutividade hidráulica saturada - $\mathbf{k}_{0}$, porosidade drenável - PD e conteúdo de água volumétrico na capacidade de campo - $\theta_{\text {cc }}$ ), na bacia hidrográfica do Ribeirão Marcela, representativa do domínio dos Latossolos na Região Alto Rio Grande, ajustando-se semivariogramas do tipo esférico e exponencial pelos métodos dos Quadrados Mínimos Ponderados (QMP) e Máxima Verossimilhança (MV). As amostras para caracterização físico-hídrica foram coletadas na camada de 0 a $0,15 \mathrm{~m}$ de profundidade, obedecendo a grids de $240 \times 240 \mathrm{~m}$ e $60 \times 60 \mathrm{~m}$, totalizando 165 pontos amostrais. Os atributos VTP e $\theta_{\text {cc }}$ apresentaram baixa variabilidade, com coeficiente de variação $(\mathrm{CV})$ de 7,54 e $10,22 \%$, respectivamente, sendo a variabilidade do atributo PD média, com CV de 43,2 \%, e alta para $\mathrm{k}_{0}$, com $\mathrm{CV}$ de $88,37 \%$. Todos os atributos apresentaram estrutura de dependência espacial, com forte grau para os atributos VTP, $\mathrm{k}_{0}$ e $\theta_{\text {cc }}$, com GD de 81,82, 75,31 e 82,61\%, respectivamente, e moderada para PD, com GD na ordem de $54,88 \%$. Os alcances para VTP, $\theta_{\text {cc }}$ e PD foram da ordem de $1.000,0 \mathrm{~m}$, enquanto o atributo $\mathrm{k}_{0}$ mostrou alcance de $77,46 \mathrm{~m}$. Verificou-se desempenho similar entre os métodos empregados, sendo ambos indicados para ajuste de semivariogramas a atributos físico-hídricos do solo. Entre os modelos de semivariograma, baseando-se na autovalidação, o esférico seria indicado para os atributos VTP, $\mathrm{k}_{0}$ e $\boldsymbol{\theta}_{\mathrm{cc}}$, apresentando quadrado médio do erro na ordem de 0,00161; 0,2795 e 0,00155, respectivamente; e o exponencial, para o atributo

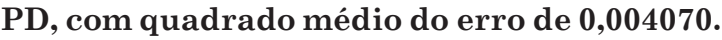

Termos de indexação: física do solo, geoestatística, bacia hidrográfica autovalidação.

\footnotetext{
(1) Parte da Tese de Mestrado do primeiro autor apresentada à Universidade Federal de Lavras - UFLA Financiado pelo Projeto de Pesquisa e Desenvolvimento 076 (P\&D 076 - CEMIG/ANEEL). Recebido para publicação em janeiro de 2006 e aprovado em fevereiro de 2007.

${ }^{(2)}$ Professor da Escola Agrotécnica Federal Antonio José Teixeira - EAFAJT. Guanambi (BA). Bolsista do CNPq. E-mail: natalagricola@yahoo.com.br

(3) Professor da Universidade Federal de Lavras - UFLA. Bolsista CNPq. E-mail: marciano@ufla.br; crmello@ufla.br; mafaria@ufla.br

(4) Pesquisadora Dra., DAG/UFLA. Bolsista Embrapa/Café. E-mail: polyanna.oliveira@uol.com.br
} 


\title{
SUMMARY: FITTINGMETHODSAND SEMI-VARIOGRAMMODELSAPPLIED TO THE STUDY OF SPATIAL VARIABILITY OF PHYSICAL- HYDRIC SOIL ATTRIBUTES
}

\begin{abstract}
The spatial variability and spatial dependence structure of physical water soil attributes (total porosity - VTP, saturated hydraulic conductivity - $k_{0}$, drainable porosity $D P$ and soil moisture at field capacity - $\theta c c)$ was evaluated for an Oxisol representative of Ribeirão Marcela watershed, in the Alto Rio Grande domain. Spherical and exponential semi-variograms were fitted by the weighted minimum square (WMS) and maximum likelihood (ML) methodologies. Undisturbed and disturbed physical water samples were collected from the 0-0.15 m layer for characterization, in grids of $240 \times 240 \mathrm{~m}$ and $60 \times 60 \mathrm{~m}$, totaling 165 sampling points. VTP and $\theta c c$ presented low variability, with coefficients of variation $(C V)$ of $7.54 \%$ and $10.22 \%$, respectively. The mean DP variability was intermediate, with $C V$ of $43.2 \%$, and was high for $k_{0}$ with a $C V$ of $88.3 \%$. All attributes presented spatial dependence structure, which was strong for VTP, $\theta c c$ and $k_{0}$, with a dependence degree $(D D)$, respectively, of $81.82,75.31$ and $82.61 \%$. The spatial dependence structure of DP was moderate with a DD of $54.66 \%$. The domains of VTP, qcc and DP were around $1.000 \mathrm{~m}$ and for $k_{0}$ it was about $77.46 \mathrm{~m}$. The results of both methodologies were similar, so that both are recommendable for the fitting of semi-variograms to physical water soil attributes. Based on cross-validation, spherical semi-variograms can be indicated for VTP, qcc and $k_{0}$, with a Mean Square Error (MSE) close to 0.00161, 0.2795 and 0.00155, respectively, and the exponential model recommended for DP, with MSE of 0.004070.
\end{abstract}

Index terms: Soil physics, geostatistics, watershed, cross-validation.

\section{INTRODUÇÃO}

A qualidade estrutural dos solos tem sido associada às condições favoráveis ao crescimento radicular, aeração, infiltração e movimento de água no seu perfil. Dentre os fatores que afetam essa qualidade, pode-se destacar o manejo empregado no cultivo e na colheita (Cichota et al., 2003), além das próprias condiç̃es de formação do solo, que lhe conferem variabilidade natural (Souza et al., 2004).

As principais alterações são evidenciadas pelo aumento da densidade do solo e pela redução do teor de matéria orgânica, tendo como conseqüência o aumento da resistência à penetração radicular, a redução a aeração e a modificação na disponibilidade e fluxo de água (Camargo \& Alleoni, 1997).

Entre os atributos físico-hídricos do solo, aqueles que se relacionam com a dinâmica da água, como conteúdo de água volumétrico na capacidade de campo $\left(\theta_{\text {cc }}\right)$, porosidade drenável (PD), em especial a condutividade hidráulica $\left(\mathrm{k}_{0}\right)$ e o volume total de poros (VTP), apresentam grande variabilidade, mesmo em solos com aparente homogeneidade (Machado,1994).

Eguchi et al. (2003) relatam coeficiente de variação (CV) de 61,28\% para a $\mathrm{k}_{0}$, com moderada estrutura de dependência espacial. No trabalho de Nielsen et al. (1973), em uma área de 150 ha com 120 pontos de amostragem, foi obtido CV de $17 \%$ para retenção de água. Esse valor difere do encontrado por Souza et al. (2004), que relatam CV de 8,68 \% para teor de água no solo, com elevado grau de dependência (90\%).
Queiroz et al. (1999) estudaram a variabilidade espacial da porosidade drenável (PD) em um solo de várzea, encontrando média variabilidade $(\mathrm{CV}=$ 43,8 \%), além de fraca dependência espacial.

A utilização da geoestatística permite identificar a existência ou não de dependência espacial entre as observações, podendo ser aplicada em mapeamentos, orientação de futuras amostragens e modelagens, permitindo, assim, estimar o valor do atributo em locais não amostrados, facilitando a gestão dos recursos naturais, especialmente em bacias hidrográficas representativas no contexto pedológico e de uso do solo.

Estudando a otimização amostral de atributos de Latossolos considerando aspectos solo-relevo, Souza et al. (2006) relatam que a utilização do alcance do semivariograma diminuiu o número de amostras a serem coletadas para avaliação de atributos químicos como Ca, P, K, CTC e saturação por bases, quando comparados à fórmula de Cline. Relatam ainda que, para garantir a dependência espacial, os pontos amostrais devem ser coletados a uma distância equivalente à metade do alcance. Motomiya et al. (2006) usaram a krigagem indicatriz na avaliação de indicadores de fertilidade do solo e constataram a possibilidade de identificar regiões com diferentes níveis de fertilidade do solo na área experimental, o que possibilita um programa de manejo regionalizado do solo, seguindo princípios da agricultura de precisão, ou seja, a aplicação de insumos e práticas agrícolas de acordo com as necessidades específicas de cada local da área. 
Objetivou-se com este trabalho identificar o melhor modelo geoestatístico para o estudo da variabilidade espacial dos atributos físico-hídricos do solo, sendo eles: volume total de poros, condutividade hidráulica saturada, porosidade drenável e teor de água na capacidade de campo na camada de 0 a $0,15 \mathrm{~m}$, em uma bacia hidrográfica representativa do domínio dos Latossolos na Região Alto Rio Grande, empregandose técnicas de estatística descritiva e procedimentos geoestatísticos na análise dos dados.

\section{MATERIAL E MÉTODOS}

A área de estudo, com aproximadamente 477 ha, compreende a sub-bacia hidrográfica do Ribeirão Marcela, localizada na margem direita do Córrego Jaguara, que deságua diretamente no reservatório da UHE de Camargos/CEMIG, município de São João Del Rei-MG. Situa-se entre as coordenadas UTM "550169" e "552810" de longitude W e "7650163" e "7650989" de latitude S e entre as altitudes de 960 e $1.060 \mathrm{~m}$, estando inserida na unidade geomorfológica Planalto Campos das Vertentes, Alto Rio Grande, Minas Gerais.

As unidades pedológicas presentes na bacia hidrográfica são Latossolo Vermelho-Amarelo (LV), ocupando aproximadamente $65 \%$ da área; Latossolo Vermelho distrófico típico (LVd), em $14 \%$; Cambissolos (C), menos de $5 \%$; e solos Hidromórficos (Hi), cerca $17 \%$. Nessa bacia hidrográfica predominam pequenos estabelecimentos rurais, voltados quase que exclusivamente para a produção leiteira, principal atividade econômica da região, com pastagem plantada e natural (362,36 ha). Entretanto, são observadas lavouras de milho (79,73 ha), maracujá (1,73 ha), cana-de-açúcar (1,53 ha), eucalipto (9,13 ha), área inundada por lago (3,27 ha) e alguns fragmentos de mata natural (19,83 ha).

O clima, segundo classificação de Köppen, é do tipo Cwa (Giarola et al., 1997). A vegetação dominante é o Cerrado tropical subcaducifólio, ocorrendo ocasionalmente floresta tropical subcaducifólia ou campo de surgente no terço inferior das encostas, campo Cerrado tropical nos locais de domínio de solos mais rasos e campo hidrófilo de várzea nas baixadas.

As amostras para caracterização físico-hídrica foram coletadas obedecendo a grids localizados por conveniência de 240 × 240 m (80 pontos, macroescala) e 60 x 60 m (79 pontos, microescala) e mais outros seis pontos aleatoriamente, com a finalidade de detalhar uma região sob vegetação de Cerrado mais densa. Ao todo, foram amostrados 165 pontos.

A razão para se fazer um detalhamento em escala menor é a possibilidade de alta variabilidade dos atributos físico-hídricos do solo, na amostragem em pequena escala. Essa variabilidade pode dificultar a estruturação de semivariogramas, devido à capacidade infinita de variação, ou seja, a não-estacionariedade de segunda ordem para grandes distâncias (Vieira, 2000). Além disso, a amostragem em pequena escala é fundamental para redução do efeito pepita dos semivariogramas, que dizem respeito a erros dos quais não se tem controle (erros aleatórios). A cobertura vegetal e as unidades pedológicas foram utilizadas como critério para locação dos pontos amostrados em pequena escala.

As amostras para caracterização físico-hídrica foram coletadas na camada de 0 a $0,15 \mathrm{~m}$ de profundidade, seguindo as recomendações para cada atributo, de acordo com Dias Júnior et al. (2000). O VTP foi calculado segundo método proposto por Embrapa (1997); a PD foi considerada como a diferença entre VTP e $\theta_{\text {cc }}$, como descrito em Mello et al. (2002). A $\theta_{\text {cc }}$ foi considerada como o teor de água de equilíbrio em amostras indeformadas, submetidas às tensões de sucção de $10 \mathrm{kPa}$, conforme Medina \& Oliveira Jr (1987). As medidas de $\mathrm{k}_{0}$ foram obtidas utilizando-se o permeâmetro de fluxo constante (permeâmetro de Ghelph - modelo 2800KI), seguindo procedimentos descritos por Eguchi et al. (2003) e Guimarães (2000).

Os dados foram submetidos, primeiramente, a uma análise exploratória, a qual consistiu de histogramas das diferenças entre pontos amostrados, média, coeficiente de variação (CV), coeficiente de assimetria (AC) e verificação de tendências com gráficos nas direções N-S (latitude) e E-W (longitude), sendo este último estudo fundamental para a aplicação das teorias geoestatísticas. Além disso, gráficos boxplot para remoção de outliers - os quais se constituem de dados que promovem aumento da variabilidade, sendo fruto de equívocos na sua obtenção no campo ou laboratório - foram desenvolvidos.

A partir da análise exploratória dos dados, a geoestatística foi utilizada para estudar a variabilidade espacial, bem como para escolha do modelo de semivariograma que melhor descrevesse a variabilidade deles.

Os valores de semivariância foram obtidos por meio da equação:

$$
\gamma^{*}(h)=\frac{1}{2 N(h)} \sum_{i=1}^{N(h)}[Z(x i)-Z(x i+h)]^{2}
$$

em que N(h) é o número de pares de valores medidos $\mathrm{Z}(\mathrm{xi}), \mathrm{Z}(\mathrm{xi}+\mathrm{h})$, separados por um vetor $\mathrm{h}$. O gráfico de $\gamma^{*}(\mathrm{~h})$ em função dos valores correspondentes de $h$, chamado semivariograma, é uma função do vetor h e, portanto, depende exclusivamente da distância $h$.

Após o cálculo das semivariâncias, foram ajustados semivariogramas (esférico e exponencial) pelos métodos dos quadrados mínimos ponderados (QMP) e máxima verossimilhança (MV) (Mello, 2004).

$$
\gamma(\mathrm{h})=\mathrm{C}_{0}+\mathrm{C}_{1}\left[\frac{3}{2}\left(\frac{\mathrm{h}}{\mathrm{a}}\right)-\frac{1}{2}\left(\frac{\mathrm{h}}{\mathrm{a}}\right)^{3}\right] ; 0<\mathrm{h}<\mathrm{a}
$$




$$
\gamma(\mathrm{h})=\mathrm{C}_{0}+\mathrm{C}_{1}\left[1-\exp \left(-3 \frac{\mathrm{h}}{\mathrm{a}}\right)\right] ; \mathrm{a}<\mathrm{h}<\mathrm{d}
$$

sendo h: distância máxima em que o semivariograma é definido; $\mathrm{C}_{0}$ : efeito pepita (ponto de interseção da curva com o eixo y); $\mathrm{C}_{1}$ : patamar; e a: alcance do semivariograma.

$\mathrm{Na}$ escolha do melhor método e modelo de ajuste foram consideradas as seguintes avaliações:

(a) Grau de dependência espacial segundo Robertson (1998):

$$
\mathrm{GD}=\left(\frac{\mathrm{C}_{1}}{\mathrm{C}_{1}+\mathrm{C}_{0}}\right) \times 100
$$

sendo o maior GD indicativo de um modelo com maior estrutura de continuidade espacial.

(b) Estudo do erro médio, máximo e quadrado médio do erro das estimativas feitas pela krigagem geoestatística, segundo procedimentos de autovalidação (validação cruzada), a qual foi realizada para todos os modelos de semivariograma e métodos de ajuste trabalhados, uma vez que a krigagem está fortemente associada ao modelo de semivariograma escolhido, permitindo que a autovalidação possa ser usada para tomada de decisão sobre o modelo e método a serem aplicados (Mello, 2004).

O programa geoR foi utilizado em todas as análises do presente trabalho, seguindo instruções de Ribeiro Junior \& Diggle (2001) e Mello (2004).

\section{RESULTADOS E DISCUSSÃO}

\section{Análise exploratória dos dados}

Observa-se que o comportamento dos dados nas direções N-S (latitude) e E-W (longitude) tem boa distribuição espacial, ou seja, não se verificam tendências à medida que se caminha nestas direções, em todos os atributos estudados (Figura 1). A existência de tendenciosidade poderia inviabilizar a aplicação da geoestatística, uma vez que a condição de não-tendenciosidade é de fundamental importância, sendo exigida para aplicação de seus princípios. É também requerida para aplicação da hipótese intrínseca, constituindo-se em um fator influenciador da estrutura de dependência espacial.

Os pontos que produzem alta variabilidade aos dados e distorção destes em torno da média, prejudicando sua normalidade, denominados outliers, podem ser vistos nos gráficos boxplot. Consta também na figura 1 o comportamento dos histogramas de freqüências das diferenças entre os pontos amostrados; a existência de normalidade dessas diferenças é de fundamental importância em estudos que utilizam os algoritmos de máxima verossimilhança, a qual consiste da aplicação de um modelo multivariado normal (Ribeiro Junior \& Diggle, 2001).

Constata-se que há candidatos a outliers, o que certamente prejudicaria a análise dos dados, caso não fossem removidos. Observa-se apenas um candidato a outlier para o atributo VTP (letra a), assim como existência de normalidade das diferenças, mostrada pelo histograma de freqüência. $\mathrm{O}$ atributo $\mathrm{k}_{0}$ (letra $\mathrm{b}$ ) apresentou oito candidatos a outlier, sendo o atributo com maior número destes.

Farias (1999) enfatiza que $\mathrm{k}_{0}$ possui alta variabilidade $(153,55 \%<\mathrm{CV}<228,92 \%)$ e não apresenta estrutura de dependência espacial definida. Assim, é possível observar que após a remoção de outiliers esse atributo apresentou considerável normalidade das diferenças, significando boa possibilidade de ajuste do semivariograma pelo algoritmo da MV. Os atributos PD e $\theta_{c c}$ não apresentaram candidatos a outlier, notando-se boa distribuição de freqüências em termos de normalidade, com o gráfico boxplot acusando boa distribuição dos dados para o atributo $\theta_{\mathrm{cc}}$ e ligeira distorção para PD.

Constatou-se pequena variabilidade dos dados, exceto para o atributo $\mathrm{k}_{0}$, dada pelos baixos coeficientes de variação (Quadro 1). Essa variabilidade pode ser classificada de acordo com os critérios propostos por Warrick \& Nielsen (1980), os quais consideram os valores do coeficiente de variação entre 12 e $60 \%$ como de média variabilidade, e os valores abaixo e acima deste intervalo, como de baixa e alta variabilidade, respectivamente.

Assim, os atributos VTP e $\theta_{\mathrm{cc}}$ apresentaram baixa variação, enquanto $\mathrm{PD}$ e $\mathrm{k}_{0}$, média e alta variação, respectivamente. Todos os atributos, mostraram coeficiente de assimetria menor que 1, significando alguma tendência à normalidade, especialmente VTP e $\theta_{\text {cc }}$, com o maior valor para $\mathrm{k}_{0}$. No entanto, a geoestatística trabalha com a diferença entre pares de pontos e não com os dados em si, requerendo então a normalidade dessas diferenças para ajuste de semivariogramas pelo algoritmo de máxima verossimilhança. Os histogramas de freqüência (Figura 1) permitem inferir que há normalidade das diferenças entre os dados, esperando-se bom ajuste dos semivariogramas pelo método da máxima verossimilhança, pelo fato de o ajuste do modelo de semivariograma por este método não se basear nos pontos do semivariograma e sim nas diferenças dos pares de pontos, sendo requisito a existência de normalidade multivariada (Diggle \& Ribeiro Junior, 2000). 
(a) Volume total de poros $\left(\mathrm{cm}^{3} \mathrm{~cm}^{-3}\right)$
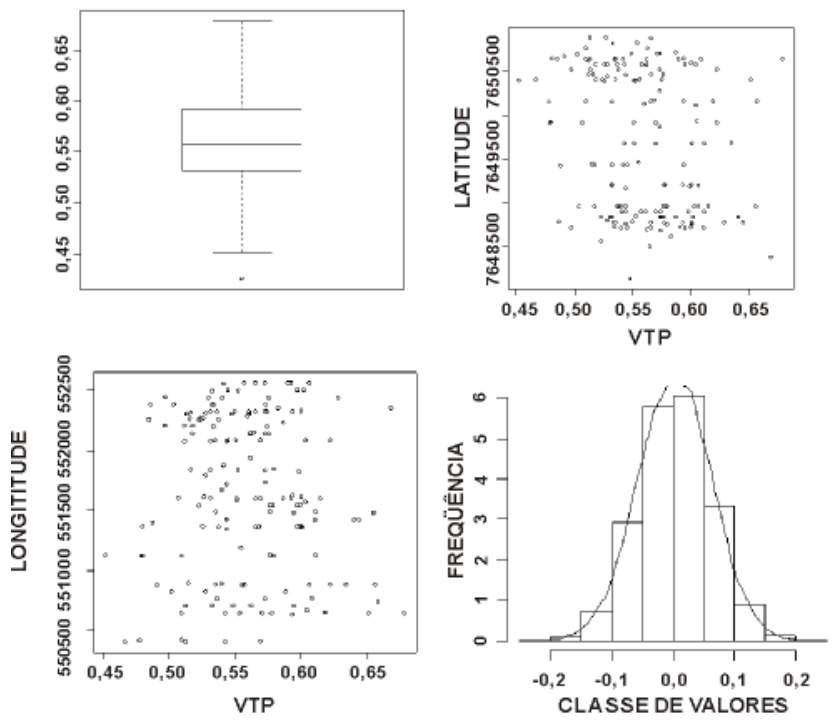

(c) Porosidade drenável - PD $\left(\mathrm{cm}^{3} \mathrm{~cm}^{-3}\right)$
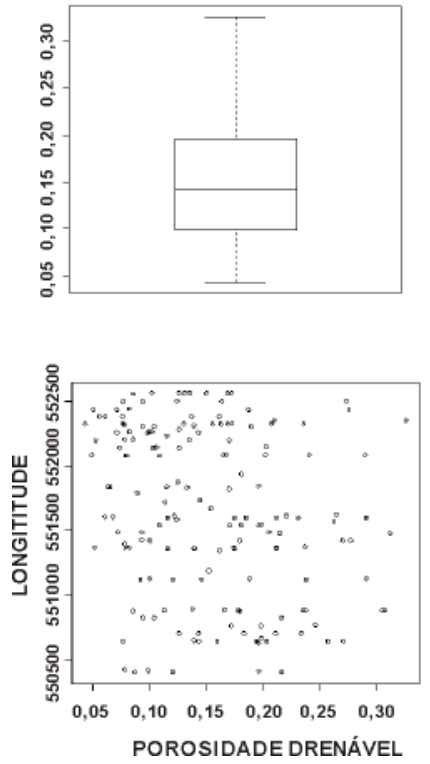

CLASSE DE VALORES (b) Condutividade hidráulica $-\mathbf{k}_{0}\left(\mathrm{~m} \mathrm{dia}^{-1}\right)$
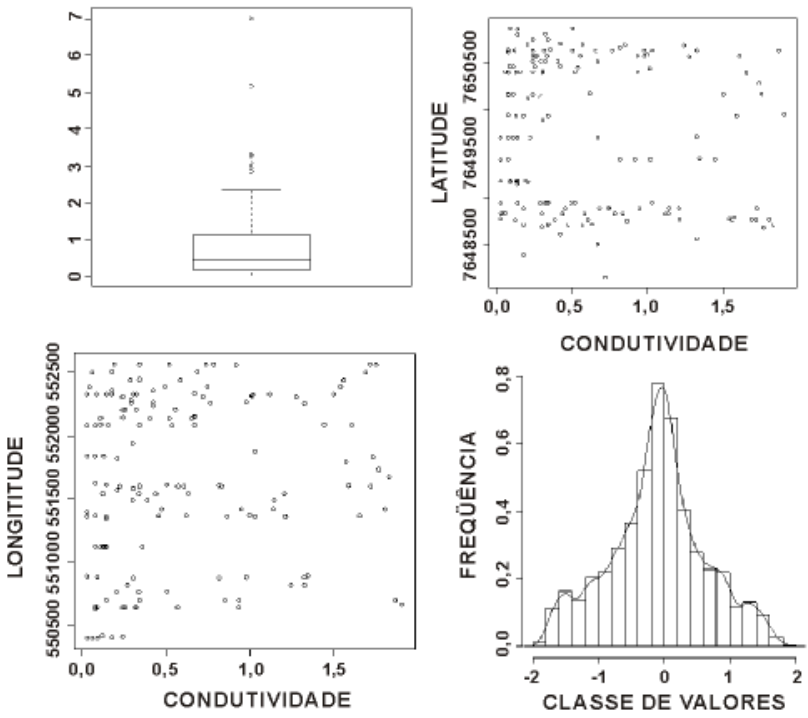

(d) $\theta_{\mathrm{Ce}}\left(\mathrm{cm}^{3} \mathrm{~cm}^{-3}\right)$
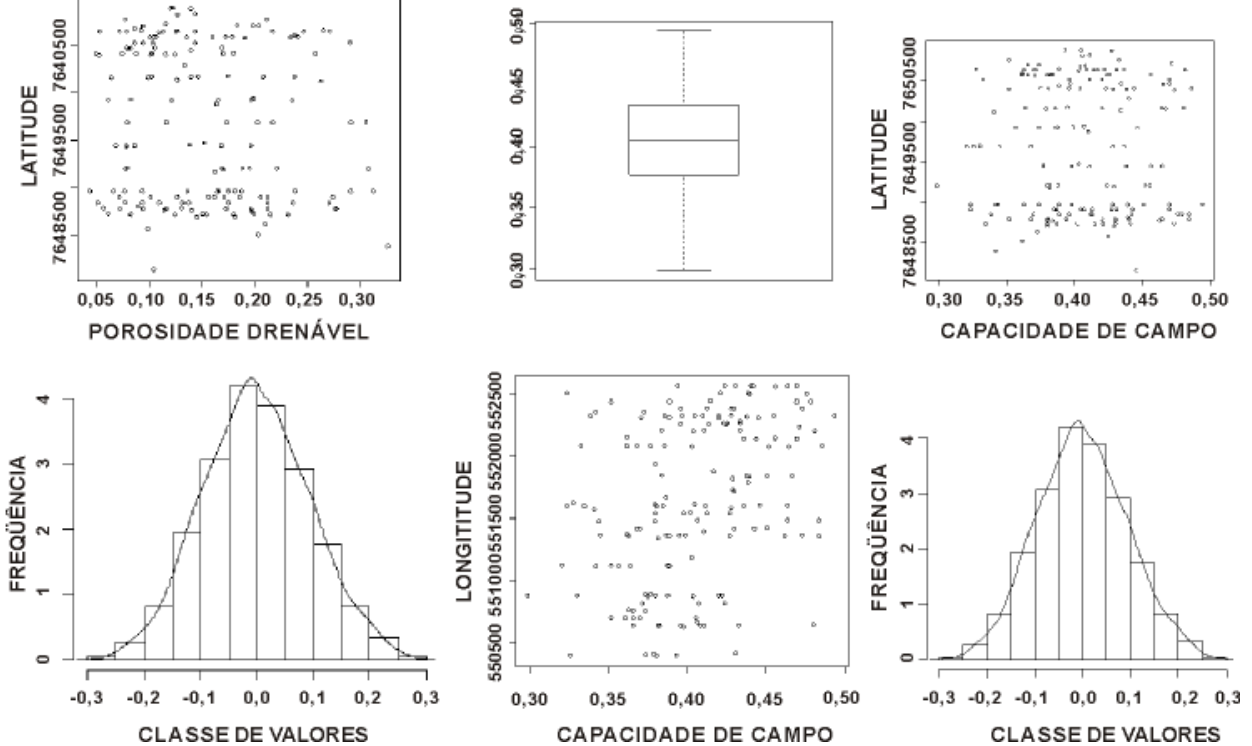

Figura 1. Gráficos da análise exploratória dos dados (boxplot, tendências e histograma da diferença entre pares de pontos) para os atributos: (a) VTP, (b) $\mathrm{k}_{0}$, (c) $\mathrm{Pd}$ e (d) $\theta_{\mathrm{cc}}$.

Quadro 1. Estatística básica dos atributos físicos volume total de poros (VTP), condutividade hidráulica saturada $\left(k_{0}\right)$, porosidade drenável (PD) e teor de água volumétrico na capacidade de campo $\left(\theta_{\text {cc }}\right)$

\begin{tabular}{lccc}
\hline Variável & CV (\%) & CA & Média \\
\hline VTP $\left(\mathrm{cm} \mathrm{cm}^{-3}\right)$ & 7,54 & 0,191 & 0,559 \\
$\mathrm{~K}_{0}\left(\mathrm{~m} \mathrm{dia}^{-1}\right)$ & 88,37 & 0,970 & 0,598 \\
PD $\left(\mathrm{cm}^{3} \mathrm{~cm}^{-3}\right)$ & 43,20 & 0,517 & 0,153 \\
$\theta_{\mathrm{CC}}\left(\mathrm{cm}^{3} \mathrm{~cm}^{-3}\right)$ & 10,22 & $-0,033$ & 0,406 \\
\hline
\end{tabular}

VTP: volume total de poros, $\mathrm{k}_{0}$ : condutividade hidráulica saturada, PD: porosidade drenável e $\theta_{\mathrm{cc}}$ : teor de água volumétrico na capacidade de campo. 


\section{Análise do ajuste de semivariogramas}

Percebe-se que os modelos ajustados por MV apresentaram semelhança em todos os atributos estudados (Figura 2). A partir da distância aproximada de $550,1.500$ e $1.200 \mathrm{~m}$, para os atributos $\mathrm{k}_{0}$, $\mathrm{PD}$ e $\theta_{\mathrm{cc}}$, respectivamente, as curvas se confundem; já para o atributo VTP, as curvas mantêm-se próximas, mas não se tangenciam, mostrando comportamento semelhante entre si.

Essa mesma análise, para os modelos ajustados por QMP, revela também que há semelhanças entre eles, exceção feita ao ajuste para $\theta_{c c}$; contudo, este método não depende da existência de normalidade multivariada, não afetando o ajuste e sim a distribuição dos pontos no semivariograma e a quantidade de diferenças que constituem cada ponto, formando os pesos de ajuste.

Ao comparar os respectivos modelos ajustados por MV e QMP, observam-se grandes discrepâncias entre os ajustes, ou seja, em nenhum atributo, os respectivos modelos apresentaram similaridade entre os métodos, sugerindo que possa haver melhor método de ajuste, o qual produzirá um mapa de krigagem mais consistente, representativo da realidade física da bacia hidrográfica.
O grau de dependência espacial é um bom indicador de um possível melhor método. Assim, observa-se melhor desempenho do método da máxima verossimilhança (MV) para o atributo $\mathrm{k}_{0}$, sobressaindo, para os demais, o método dos quadrados mínimos ponderados (QMP) (Quadro 2). O melhor desempenho deste método pode ser atribuído ao menor efeito pepita, em comparação ao MV, conjuntamente à existência de um patamar de mesma magnitude.

Outra observação importante referente aos semivariogramas ajustados para o atributo $\mathrm{k}_{0}$ é que o modelo exponencial ajustado por QMP produziu efeito pepita puro, sendo o único ajuste dessa natureza. Esse fato reflete a alta variabilidade apresentada por este atributo, bem como as possíveis interações entre $\mathrm{k}_{0}$, VTP e PD. A ocorrência do efeito pepita puro permite inferir que as amostras para estudo de $\mathrm{k}_{0}$, nesta subbacia hidrográfica, devem ser feitas a distâncias menores que as utilizadas na amostragem deste trabalho. De maneira geral, o método QMP apresentou melhor desempenho, com os modelos mostrando baixo efeito pepita e maior grau de dependência espacial.

Tomando-se como base os valores do grau de dependência espacial sugeridos por Cambardella et al. (1994), há forte grau de dependência (> $75 \%$ ) para VTP e $\theta_{\text {cc }}$, considerando o modelo esférico ajustado por

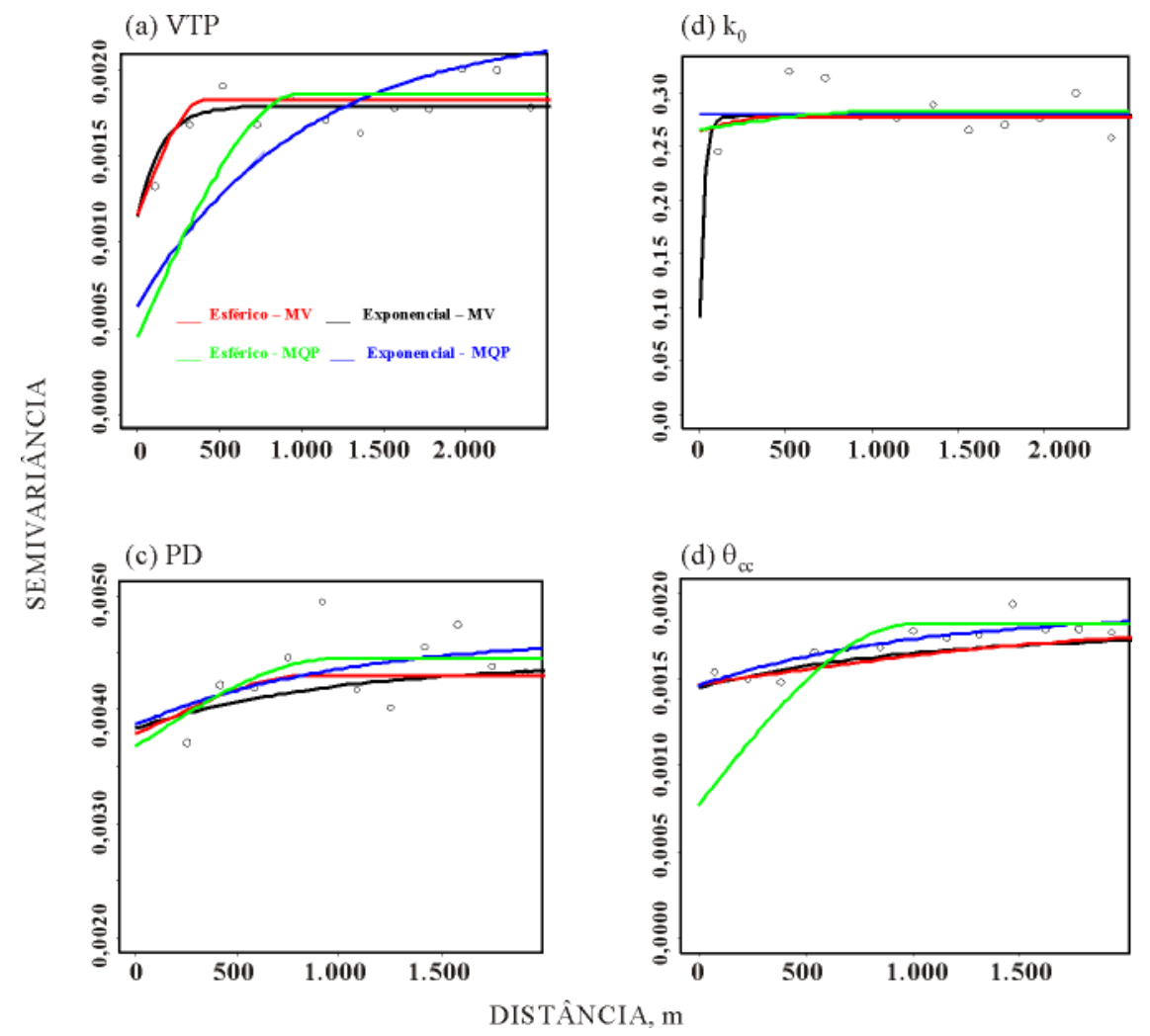

Figura 2. Comportamento dos modelos de semivariograma esférico e exponencial ajustados pelos métodos da MV e QMP, para os atributos: (a) VTP, (b) $\mathrm{k}_{0}$, (c) PD e (d) $\theta_{\text {cc }}$ 
Quadro 2. Parâmetros de ajuste dos modelos de semivariograma exponencial e esférico, ajustados por máxima verossimilhança (MV) e quadrados mínimos ponderados (QMP), e os respectivos graus de dependência espacial

\begin{tabular}{|c|c|c|c|c|c|c|}
\hline \multirow{2}{*}{ Atributo } & \multirow{2}{*}{ Modelo } & \multirow{2}{*}{ Método } & \multicolumn{3}{|c|}{ Parämetro } & \multirow{2}{*}{ GD } \\
\hline & & & Efeito pepita & Patamar & Alcance & \\
\hline & & & & & $\mathrm{m}$ & $\%$ \\
\hline \multirow[t]{4}{*}{ VTP } & Exponencial & MV & 0,0012 & 0,0006 & 436,56 & 60,00 \\
\hline & Esférico & MV & 0,0012 & 0,0007 & 406,75 & 61,29 \\
\hline & Exponencial & QMP & 0,0006 & 0,0016 & 3000,00 & 78,57 \\
\hline & Esférico & $\mathrm{QMP}$ & 0,0004 & 0,0014 & 1000,00 & 81,82 \\
\hline \multirow[t]{4}{*}{$\mathrm{K}_{0}$} & Exponencial & MV & 0,0909 & 0,1873 & 77,46 & 75,31 \\
\hline & Esférico & MV & 0,2644 & 0,0135 & 436,19 & 51,24 \\
\hline & Exponencial $^{(1)}$ & QMP & 0,2802 & - & - & - \\
\hline & Esférico & $\mathrm{QMP}$ & 0,2657 & 0,0164 & 1000,00 & 51,50 \\
\hline \multirow[t]{4}{*}{$\mathrm{PD}$} & Exponencial & MV & 0,0038 & 0,0006 & 3000,00 & 53,66 \\
\hline & Esférico & MV & 0,0038 & 0,0005 & 887,95 & 53,09 \\
\hline & Exponencial & QMP & 0,0039 & 0,0008 & 3000,00 & 54,65 \\
\hline & Esférico & $\mathrm{QMP}$ & 0,0037 & 0,0008 & 1000,00 & 54,88 \\
\hline \multirow[t]{4}{*}{$\theta_{\mathrm{CC}}$} & Exponencial & MV & 0,0015 & 0,0003 & 3000,00 & 54,55 \\
\hline & Esférico & MV & 0,0015 & 0,0003 & 2362,60 & 54,55 \\
\hline & Exponencial & $\mathrm{QMP}$ & 0,0015 & 0,0004 & 3000,00 & 55,88 \\
\hline & Esférico & $\mathrm{QMP}$ & 0,0008 & 0,003 & 1000,00 & 82,61 \\
\hline
\end{tabular}

(1) Efeito pepita puro, VTP: volume total de poros, em $\mathrm{cm}^{3} \mathrm{~cm}^{-3} ; \mathrm{k}_{0}$ : condutividade hidráulica saturada, em $\mathrm{m} \mathrm{dia}{ }^{-1}$; PD: porosidade drenável, em $\mathrm{cm}^{3} \mathrm{~cm}^{-3}$; e $\theta_{\mathrm{cc}}$ : umidade volumétrica na capacidade de campom $\mathrm{em}^{3} \mathrm{~cm}^{-3}$.

QMP, assim como para $\mathrm{k}_{0}$, com o modelo exponencial por MV. Em se tratando de PD, o grau de dependência é moderado para ambos os modelos e métodos empregados.

$\mathrm{A}_{0}$ de um solo é determinada pela geometria e continuidade dos poros preenchidos com água, tornando-se dependente, portanto, da forma, quantidade, distribuição e continuidade destes; assim, é de se esperar que a $\mathrm{k}_{0}$ de $\mathrm{um}$ solo que apresenta atributos como VTP e PD bem estruturados espacialmente herde algumas características, fato este que ajuda a explicar a forte dependência espacial obtida para este atributo. A literatura relata alguns métodos indiretos para obtenção de $\mathrm{k}_{0}$ utilizando variáveis nãohidráulicas do solo, como densidade do solo, porosidade total, macro e microporosidade, as quais influenciam a distribuição dos poros e, por conseqüência, $a \mathrm{k}_{0}$. Mesquita (2001) relata que a variabilidade encontrada nas medidas da macroporosidade - evidenciadas principalmente pelo coeficiente de variação, geralmente, variando de 15 a $20 \%$ - condiciona alta variabilidade em $\mathrm{k}_{0}$, o que também pode ser confirmado pelo coeficiente de variação elevado de $\mathrm{k}_{0}$, geralmente maior que $70 \%$, o que vai ao encontro dos resultados obtidos.
São visualizados no quadro 3 os valores dos erros médio e máximo, bem como a média do quadrado médio do erro gerado pela autovalidação, ou seja, comparando os valores preditos pela krigagem com os valores observados.

Observa-se que não há predominância de um modelo de semivariograma, mas, quando se analisa o método, fica evidente o melhor desempenho do algoritmo de máxima verossimilhança, o que vem de encontro ao comportamento dos histogramas apresentados na figura 1 , ou seja, normalidade multivariada das diferenças entre pares de pontos.

Essas informações permitem inferir, com maior acurácia, o desempenho dos modelos e métodos empregados. Num primeiro instante, a grandeza dos valores dos erros médio e máximo para $\mathrm{k}_{0}$ chama a atenção, quando comparados aos erros dos demais atributos. Isso é reflexo da alta variabilidade deste atributo, como anteriormente discutido, e está relacionado a um ajuste de semivariograma de qualidade inferior aos obtidos para os demais atributos, tendo em vista as dificuldades estatísticas apresentadas pelos dados, comentadas anteriormente. Comportamento semelhante pode ser observado para PD, o qual também apresenta alta variabilidade. 
Quadro 3. Erro médio (EM), máximo (EMa) e quadrado médio do erro (QME), gerados pela autovalidação, para os respectivos modelos e métodos de ajuste

\begin{tabular}{|c|c|c|c|c|c|}
\hline Atributo & Modelo & Método & EM & E. Ma & QME \\
\hline & & & -1 & 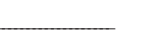 & \\
\hline \multirow[t]{4}{*}{ VTP } & Exponencial & MV & 5,88 & 22,149 & 0,001650 \\
\hline & Esférico & MV & 5,84 & 21,168 & 0,001610 \\
\hline & Exponencial & QMP & 5,89 & 20,720 & 0,001670 \\
\hline & Esférico & QMP & 5,99 & 20,736 & 0,001710 \\
\hline \multirow[t]{4}{*}{$\mathrm{K}_{0}$} & Exponencial & MV & 238,27 & 2136,0 & 0,279000 \\
\hline & Esférico & MV & 235,24 & 2110,0 & 0,279500 \\
\hline & Exponencial $^{(1)}$ & QMP & - & - & - \\
\hline & Esférico & QMP & 234,09 & 2061,0 & 0,278000 \\
\hline \multirow[t]{4}{*}{$\mathrm{PD}$} & Exponencial & MV & 43,48 & 226,63 & 0,004070 \\
\hline & Esférico & MV & 43,69 & 217,90 & 0,004100 \\
\hline & Exponencial & QMP & 43,34 & 223,59 & 0,004070 \\
\hline & Esférico & QMP & 43,40 & 214,89 & 0,004110 \\
\hline \multirow[t]{4}{*}{$\theta_{\mathrm{CC}}$} & Exponencial & MV & 8,00 & 31,47 & 0,001556 \\
\hline & Esférico & MV & 7,96 & 31,12 & 0,001550 \\
\hline & Exponencial & QMP & 8,00 & 32,03 & 0,001556 \\
\hline & Esférico & QMP & 8,28 & 35,84 & 0,001640 \\
\hline
\end{tabular}

(1) Efeito pepita puro; VTP: volume total de poros, em $\mathrm{cm}^{3} \mathrm{~cm}^{-3} ; \mathrm{k}_{0}$ : condutividade hidráulica saturada, em $\mathrm{m} \mathrm{dia}{ }^{-1}$; $\mathrm{m}$ : porosidade drenável, em $\mathrm{cm}^{3} \mathrm{~cm}^{-3}$; e $\theta_{\mathrm{cc}}$ : umidade volumétrica na capacidade de campo, em $\mathrm{cm}^{3} \mathrm{~cm}^{-3}$.

Com base nos valores (Quadro 3) e nas discussões já apresentadas, sugere-se, para estudo da variabilidade espacial, o ajuste pelo método da MV e modelo esférico para os atributos VTP e $\mathrm{k}_{0}$ e modelo exponencial com mesmo método para o atributo $\theta_{\text {cc. }}$. O método dos quadrados mínimos ponderados seria indicado para ajuste do modelo exponencial no estudo da variabilidade espacial do atributo $\mathrm{PD}$.

\section{CONCLUSÕES}

1. A análise exploratória de dados mostrou-se importante ferramenta no estudo de atributos físicohídricos do solo, eliminando candidatos a outliers, sendo possível observar que os atributos VTP e $\theta_{c c}$ apresentaram baixa variabilidade; o atributo PD, média variabilidade; $\mathrm{e}$ o atributo $\mathrm{k}_{0}$, alta variabilidade.

2. Verificou-se estrutura de dependência espacial para todos os atributos, sendo esta forte para VTP e $\theta_{\mathrm{cc}}$ e moderada para $\mathrm{PD}$ e $\mathrm{k}_{0}$.

3. Observou-se melhor desempenho do algoritmo de máxima verossimilhança para o ajuste de semivariogramas aplicados ao estudo da variabilidade espacial de atributos físico-hídricos do solo.

4. Com base na autovalidação, é possível sugerir o modelo esférico para os atributos VTP, $\theta_{\mathrm{cc}}$ e $\mathrm{k}_{0}$ e o exponencial para o atributo PD.

\section{LITERATURA CITADA}

CAMARGO, O.T. \& ALLEONI, L.R.F. Compactação do solo e o desenvolvimento das plantas. Piracicaba, 1997. 132p.

CAMBARDELA, C.A.; MOORMAN, T.B.; NOVAK, J.M.; PARKIN, T.B.; KERLEN, D.L.; TURCO, R.F. \& KONOPKA, A.E. Field scale variability of soil properties in Central Iowa soils. Soil Sci. Soc. Am. J., 47:1501-1511, 1994.

CICHOTA, R.; van LIER, Q.J. \& LEGUIZAMÓN ROJAS, C.A. Variabilidade espacial da taxa de infiltração em Argissolo Vermelho. R. Bras. Ci. Solo, 27:789-798, 2003.

DIAS JUNIOR, M.S.; BERTONI, J.C. \& BASTOS, A.R.R. Física do solo. Lavras, Universidade Federal de Lavras, 2000. p.147.

DIGGLE, P.J. \& RIBEIRO JÚNIOR, P.J. Model based geoestatistics. In: SIMPÓSIO NACIONAL DE PROBABILIDADE E ESTATÍSTICA, 14., Caxambu, 2000. Resumos. Caxambu, Associação Brasileira de Estatística, 2000. p.192.

EGUCHI, E.S.; SILVA, E.L. \& OLIVEIRA, M.S. Variabilidade espacial da condutividade hidráulica do solo saturado e da taxa de infiltração básica determinadas "in situ". Ci. Agrotecnol., Edição especial:1607-1613, 2003.

EMPRESA BRASILEIRA DE PESQUISA AGROPECUÁRIA EMBRAPA. Centro Nacional de Pesquisa de Solos. Manual de métodos de análise de solo. 2.ed. Rio de Janeiro, 1997. $212 \mathrm{p}$. 
FARIAS, M.S.S. Diagnóstico da necessidade de drenagem no perímetro irrigado de São Gonçalo. Campina Grande, Universidade Federal de Paraíba, 1999. 75p. (Tese de Mestrado)

GIAROLA, M.F.B.; CURI, N.; SIQUEIRA, J.O.; CHAGAS, C.S. \& FERREIRA, M.M. Solos da região sob influência do reservatório da hidrelétrica de Itutinga/Camargos (MG): perspectiva ambiental. Lavras, Convênio CEMIG/UFLA/ FAEPE, 1997. 101p.

GUIMARÃES, E.C. Variabilidade espacial de atributos de um Latossolo Vermelho-Escuro textura argilosa da região do Cerrado, submetido ao plantio direto e ao plantio convencional. Campinas, Universidade de Campinas, 2000. 85p. (Tese de Doutorado)

MACHADO, R.V. Variabilidade espacial de atributos físicohídricos em uma hidrosseqüência de solos de bem a mal drenados. Lavras, Escola Superior de Agricultura de Lavras, 1994. 88p. (Tese de Mestrado)

MEDINA, B.F. \& OLIVEIRA Jr., R.C. Relações entre capacidade de campo determinada in situ e em laboratório em Latossolo Amarelo muito argiloso. R. Bras. Ci. Solo, 11:91. 95, 1987.

MELLO, C.R..; OLIVEIRA, G.C.; RESCK, D.V.S.; LIMA, J.M. \& DIAS JUNIOR, M. S. Estimativa da capacidade de campo baseada no ponto de inflexão da curva característica. Ci. Agrotecnol., 26:837-841, 2002.

MELLO, J.M. Geoestatística aplicada ao inventário florestal. Piracicaba, Escola Superior de Agricultura Luiz de Queiroz, 2004. 110p. (Tese de Doutorado)

MESQUITA, M.G.B.F. Caracterização estatística da condutividade hidráulica saturada do solo. Piracicaba, Escola Superior de Agricultura Luiz de Queiroz, 2001. 110p. (Tese de Doutorado)
MOTOMIYA, A.V.A.; CORÁ, J.E. \& PERREIRA, C.T. Uso da krigagem indicatriz na avaliação de indicadores de fertilidade do solo. R. Bras. Ci. Solo, 30:485-496, 2006.

NIELSEN, D.R.; BIGGAR, J.W. \& ERH, K.T. Spatial variability of field measured soil water properties. Hilgardia, 42:215$259,1973$.

QUEIROZ, J.E.; CRUCIANI, D.E. \& LIBARDI, P.L. Variabilidade espacial da porosidade drenável de um solo de várzea, no município de Piracicaba, Estado de São Paulo. R. Bras. Eng. Agríc. Amb., 3:135-139, 1999.

RIBEIRO JR, P.P. \& DIGGLE, P.P. GeoR: A package for geostatistical analysis. R - News, 1:15-18, 2001.

ROBERTSON, G.P. GS ${ }^{+}$: Geostatistics for the environmental sciences - GS $^{+}$User's Guide. Plainwell, Gamma Design Soffware, 1998. 152p.

SOUZA, Z.M.; MARQUES JÚNIOR, J.; PERREIRA, G.T. \& MONTANARI, R. Otimização amostral de atributos de Latossolos considerando aspectos solo-relevo. Ci. Rural, 36:829-836, 2006.

SOUZA, Z.M.; MARQUES JÚNIOR, J.; PERREIRA, G.T. \& MOREIRA, L.F. Influência da pedoforma na variabilidade espacial de alguns atributos físicos e hídricos de um latossolo sob cultivo de cana-de-açúcar. Irriga, 9:1-11, 2004.

VIEIRA, S.R. Geoestatística em estudo de variabilidade espacial do solo. In: NOVAIS, R.F.; ALVAREZ V., V.H. \& SCHAEFER, C.E.GR., eds. Tópicos em ciência do solo. Viçosa, MG, Sociedade Brasileira de Ciência do Solo, 2000. p.1-55.

WARRICK, A.W. \& NIELSEN, D.R. Spatial variability of soil physical properties in the field. In: HILLEL, D., ed. Applications of soil physics. New York, Academic Press, 1980. p.319-344. 